\title{
The Thomas-Reiche-Kuhn Sum Rule and the Rigid Rotator
}

\author{
Evangelos Hadjimichael \\ Fairfield University, ehadjimichael@fairfield.edu
}

W. Currie

S. Fallieros

Follow this and additional works at: https://digitalcommons.fairfield.edu/engineering-facultypubs

\section{Peer Reviewed}

\section{Repository Citation}

Hadjimichael, Evangelos; Currie, W.; and Fallieros, S., "The Thomas-Reiche-Kuhn Sum Rule and the Rigid Rotator" (1997). Engineering Faculty Publications. 1.

https://digitalcommons.fairfield.edu/engineering-facultypubs/1

\section{Published Citation}

Hadjimichael, E.; Currie, W.; and Fallieros, S. 1997. The Thomas-Reiche-Kuhn Sum Rule and the Rigid Rotator. Am. J. Phys. 65, 4: 335-341.

This item has been accepted for inclusion in DigitalCommons@Fairfield by an authorized administrator of DigitalCommons@Fairfield. It is brought to you by DigitalCommons@Fairfield with permission from the rightsholder(s) and is protected by copyright and/or related rights. You are free to use this item in any way that is permitted by the copyright and related rights legislation that applies to your use. For other uses, you need to obtain permission from the rights-holder(s) directly, unless additional rights are indicated by a Creative Commons license in the record and/or on the work itself. For more information, please contact digitalcommons@fairfield.edu. 


\title{
The Thomas-Reiche-Kuhn sum rule and the rigid rotator
}

\author{
E. Hadjimichael \\ Department of Physics, Fairfield University, Fairfield, Connecticut 06430 \\ William Currie ${ }^{\text {a) }}$ and S. Fallieros \\ Department of Physics, Brown University, Providence, Rhode Island 02912
}

(Received 7 November 1995; accepted 25 September 1996)

\begin{abstract}
It is shown that the Thomas-Reiche-Kuhn sum rule, associated with the photoabsorption cross section from quantum systems, appears to be violated in the case of the quantized rigid rotator. The origin of the apparent violation is investigated, and its resolution is presented on the basis of a related system, i.e., a particle in a spherical $\delta$-function potential whose energy spectrum approaches that of the rigid rotator when the strength of the potential becomes large.
\end{abstract}

(C) 1997 American Association of Physics Teachers.

\section{INTRODUCTION}

It is well known that for relatively low energies, the interaction of electromagnetic radiation with a microscopic system is dominated by electric dipole $(E 1)$ transitions. The matrix element for the $E 1$ amplitude,

$$
E 1=\left\langle\Psi_{f}|D \cdot \hat{\epsilon}| \Psi_{i}\right\rangle,
$$

is expressed in terms of the dipole operator for the charged system, i.e.,

$$
\hat{D}=\sum_{j} e_{j} \bar{r} \delta\left(\bar{r}-\overline{r_{j}}\right),
$$

where $\hat{\epsilon}$ is the polarization of the external electric field in an arbitrary direction, and $e_{j}$ is the electric charge of the $j$ th particle. There is a well known sum rule that is associated with the E1 amplitude, the Thomas-Reiche-Kuhn sum rule, ${ }^{1}$ which we proceed to derive.

Starting from the fundamental commutation relation

$$
-\frac{i}{\hbar}\left[\hat{\epsilon}^{*} \cdot \bar{r}, \hat{\epsilon} \cdot \bar{p}\right]=1,
$$

which is intimately related to the uncertainty principle, and the identity

$$
[\hat{H}, \hat{\epsilon} \cdot \bar{r}]=-i \frac{\hbar}{m} \hat{\epsilon} \cdot \bar{p},
$$

where $\hat{H}$ is the Hamiltonian of the system under consideration, assumed to contain no velocity-dependent forces, we obtain

$$
\left\langle\Psi_{0}\left|\frac{m}{\hbar^{2}}\left[\hat{\epsilon}^{*} \cdot \hat{r},[\hat{H}, \hat{\epsilon} \cdot \hat{r}]\right]\right| \Psi_{0}\right\rangle=1,
$$

where $\Psi_{0}$ represents the ground state of the system, and $\hat{\epsilon}^{*} \cdot \hat{\epsilon}=1$. We note that for the case of linear polarization $\hat{\epsilon}$ is a real unit vector, i.e., $\hat{\epsilon}^{*}=\epsilon$, and Eq. (I5) can be expressed in Cartesian coordinates; for circular polarization, $\hat{\epsilon}^{*} \neq \hat{\epsilon}$, and Eq. (I5) would contain spherical components of the position operator $\hat{r}$, i.e., those proportional to $r Y_{l, m}(\theta, \phi)$.

By expanding the commutator in Eq. (I5) and using the closure relation for the complete set of eigenstates of the Hermitian operator $\hat{H}$, i.e.,

$$
\sum_{n} \Psi_{n}^{*}(\vec{r}) \Psi_{n}(\vec{r})=\delta(\bar{r}-\vec{r}),
$$

we finally obtain the result

$$
\sum_{n} f_{n 0}=\frac{2 m}{\hbar^{2}} \sum_{n}\left(E_{n}-E_{0}\right)\left|\left\langle\Psi_{n}|\hat{\boldsymbol{\epsilon}} \cdot \vec{r}| \Psi_{0}\right\rangle\right|^{2}=1,
$$


which is the celebrated Thomas-Reiche-Kuhn (TRK) sum rule. ${ }^{1}$ The quantities $f_{n 0}$ are the well-known "oscillator strengths," so called because of their appearance in the amplitude for scattering of light from a harmonic oscillator ${ }^{2}$ in the long wavelength (electric dipole) approximation. In Eq. (I7) we have restricted ourselves to the case of a single particle of mass $m$ bound in a potential. For a two-body system, $\mathbf{r}$ would be the separation distance between the two particles and $m$ would be replaced by the reduced mass $\mu$; for a manybody system, $\mathbf{r}$ would be replaced by a sum over particles.

As the derivation of Eq. (I7) indicates, the TRK sum rule is a direct consequence of the fundamental laws of quantum mechanics. In addition, it implies some important results concerning directly measurable quantities, such as the scattering amplitude and the cross section for photon absorption by a bound system. Before proceeding further we present here examples of such results.

It can be readily shown ${ }^{2}$ that the integrated cross section for $E 1$ photon absorption is

$$
\int_{0}^{\infty} \sigma_{\mathrm{abs}}^{E 1}(E) d E=2 \pi^{2} e^{2} \frac{\hbar}{m c} \sum_{n} f_{n 0}=2 \pi^{2} e^{2} \frac{\hbar}{m c},
$$

where the TRK sum rule was used in the last step. $\sigma_{\text {abs }}^{E 1}(E)$ is the cross section for absorption of photons of energy $E$ by a bound particle of charge $e$ in the electric dipole $(E 1)$ approximation. Equation (I8) shows that the integrated cross section has a finite upper bound independent of the nature of the binding forces. ${ }^{2-4}$

Furthermore, we find that the amplitude for forward scattering of photons of energy $E$ from a bound system, in the $E 1$ approximation, is

$$
\begin{aligned}
f_{E 1}(E)= & -E^{2} \frac{e^{2}}{\hbar c^{2}} \sum_{n}\left|\left\langle\Psi_{n}|\hat{\epsilon} \cdot \bar{r}| \Psi_{0}\right\rangle\right|^{2} \\
& \times \frac{2 E_{n 0}}{\left(E-E_{n 0}\right)^{2}+i \eta},
\end{aligned}
$$

with $E_{n 0}=E_{n}-E_{0}$ and $\eta \rightarrow 0$. It has a high energy limit

$$
f_{E 1}(E) \underset{E \rightarrow \infty}{\rightarrow}-\frac{e^{2}}{m c^{2}} \sum_{n} f_{n 0}=-\frac{e^{2}}{m c^{2}}
$$

Again, the TRK sum rule was applied in the last step. Equation (I10) indicates that at high energies, this scattering amplitude becomes identical to the Thomson amplitude describing the scattering of photons from a free particle with the same charge and mass. ${ }^{5}$

Equations (I8) and (I10) appear in many textbooks, yet a comment is in order at this point with regard to the compatibility between the long wavelength approximation which implies a low energy regime, and the high energy limit employed in these equations. It is important to remember that both equations are nonrelativistic. In this context, "high energy" implies that the energy of the photon is large compared to typical excitation or ionization energies, but still small compared to the mass of the bound particle. An atomic system is typical of this situation where the high-energy limit is still compatible with the nonrelativistic approximation and the dominance of the $E 1$ transition.

In the following we examine the saturation of the TRK sum rule, Eq. (I7), and identify the energy region which contains most of the significant oscillator strength. Thus, in Sec. II we present three typical examples which illustrate the saturation of the sum rule. In Sec. III, we consider the case of the rigid rotator which exhibits a behavior that appears at first to be idiosyncratic. In Sec. IV, we achieve an understanding of this behavior by considering the rigid rotator as the limit of a regular three-dimensional system composed of a particle bound in a spherical $\delta$-shell potential, thus avoiding the constraint of perfect rigidity. The strength of the $\delta$-shell potential that leads to the appearance of an energy spectrum approaching that of the rigid rotator is investigated. We discuss briefly some additional sum rules in Sec. V, and we present concluding remarks in Sec. VI.

\section{THREE EXAMPLES}

1. The three-dimensional harmonic oscillator The Schrödinger equation for this system is

$$
\left(\frac{\vec{p}^{2}}{2 m}+\frac{1}{2} m \omega_{0}^{2} r^{2}\right) \Psi_{n}=E_{n} \Psi_{n},
$$

and yields energy eigenvalues $E_{n}=\left(n+\frac{3}{2}\right) \hbar \omega_{0}$, so that $\left(E_{n}-E_{0}\right)=n \hbar \omega_{0}$. Furthermore, it is readily shown ${ }^{2}$ that the electric dipole matrix element is, in this case,

$$
\left\langle\Psi_{n}|\hat{\epsilon} \cdot \vec{r}| \Psi_{0}\right\rangle=\sqrt{\frac{\hbar}{2 m \omega_{0}}} \delta_{n, 1}
$$

so that $f_{10}=1$, as seen from Eq. (I7), and all the other oscillator strengths are equal to zero. The sum rule is saturated by exactly one single transition to the first excited state of the harmonic oscillator.

2. The infinite spherical well

For a particle of mass $m$ trapped in a spherical, infinitely deep, potential well of radius $R$, i.e.,

$$
V(r)=\left\{\begin{array}{lc}
0, & 0 \leqslant r \leqslant R \\
\infty, & r \geqslant R
\end{array},\right.
$$

there is an infinite number of bound states. The calculation of the oscillator strengths is straightforward and yields: ${ }^{6}$

$$
\begin{aligned}
& f_{10}=0.9676, \quad f_{20}=0.0254, \\
& f_{30}=0.0048, \quad f_{40}=0.0016, \\
& f_{50}=0.0007,
\end{aligned}
$$

with all the other oscillator strengths being negligibly small. The above numbers add up to $\sum_{n=1}^{5} f_{n 0}=1$. That is, the TRK sum rule is saturated by excitations to the first five states and is dominated by the contribution of the first excited state. Note that the indices $n=1,2,3,4,5$ in $f_{n 0}$ represent excitations of one angular momentum state, the $l=1$ state, as is clear from the nature of the electric dipole operator in Eq. (I7).

3. The hydrogen atom.

It is shown in Ref. 6 that in the case of an electron-proton system, the sum of the oscillator strengths from transitions to bound exited states amounts to

$$
\sum_{\text {bound }} f_{n 0}=0.5640 \text {, }
$$

and the equivalent sum from transitions to unbound states

$$
\sum_{\text {unbound }} f_{n 0}=0.4360
$$

so that the TRK sum rule is indeed $\Sigma_{n} f_{n 0}=1$, and is thus rigorously satisfied. The contribution from the unbound 
states in the continuum is rather well concentrated at energies not far from the ionization threshold.

\section{THE RIGID ROTATOR}

The rigid rotator is envisioned as two particles of mass $m_{1}$ and $m_{2}$, rigidly separated at a fixed distance $r=R$. Hence, this system is often used as a simplified model describing the rotational excitations of, say, a diatomic molecule. The reduced mass of the system is $\mu=m_{1} m_{2} /\left(m_{1}+m_{2}\right)$, and its moment of inertia is $I=\mu R^{2}$. The simplified Hamiltonian describing the system is $H_{R}=\overline{L^{2}} / 2 \mu R^{2}$ where $L^{2}$ is the square of the angular momentum operator which is a purely angle-dependent operator. The eigenvalues of the system are the familiar ones

$$
E_{l}=\frac{l(l+1) \hbar^{2}}{2 \mu R^{2}},
$$

where $l=0,1,2, \ldots$, while the eigenfunctions are the spherical harmonics $Y_{l, m}(\theta, \phi)$. There is only one energy state for each value of the quantum number $l$, and the only degeneracy is associated with the azimuthal quantum number $m=l, l-1, l-2 \ldots-l$. Taking the polarization $\hat{\epsilon}$ along the $z$-axis, we find the dipole matrix element to be

$$
\left\langle Y_{l, m}|\hat{\boldsymbol{\epsilon}} \cdot \vec{r}| Y_{0,0}\right\rangle=\left\langle Y_{l, m}|R \cos \theta| Y_{0,0}\right\rangle=\frac{R}{\sqrt{3}} \delta_{l, 1} \delta_{m, 0} .
$$

Evaluating the TRK sum rule, i.e., the left-hand side of Eq. (I7), by means of Eqs. (III1) and (III2) we find,

$$
\sum_{n} f_{n 0}=f_{10}=\frac{2 \mu}{\hbar^{2}}\left(\frac{2 \hbar^{2}}{2 \mu R^{2}}\right) \frac{R^{2}}{3}=\frac{2}{3} .
$$

in apparent violation of the TRK sum rule, Eq. (I7). Given the fundamental nature of this sum rule and its direct relation to physical processes as illustrated in Sec. I, the missing 1/3 in the result of Eq. (III3) deserves further discussion.

To begin to understand the result (III3), we review the derivation of Eq. (I7) using the general Hamiltonian for a particle of mass $m$,

$$
H=\frac{P_{r}^{2}}{2 m}+\frac{\overline{L^{2}}}{2 m r^{2}}+V(r)
$$

where $P_{r}=(\hat{r} \cdot \bar{p}+\bar{p} \cdot \hat{r}) / 2$ is the radial momentum of the particle in a spherically symmetric potential $V(r)$, and $\overrightarrow{L^{2}}$ is the square of the angular momentum; $\hat{r}=\vec{r} r$ is the unit vector. Equation (III4) shows explicitly the two kinetic energy terms, one associated with the radial motion and the other with rotational motion. A simple calculation shows that $1 / 3$ of the TRK sum rule originates in the $P_{r}^{2}$ term, while the remaining $2 / 3$ is obtained from the centrifugal term $\overline{L^{2}}$ even though the value of $r^{2}$ in the denominator of this term is not necessarily constant. In comparison to Eq. (III4), the Hamiltonian for the rigid rotator does not have a term proportional to the radial momentum; hence the missing $1 / 3$ in the TRK sum rule, Eq. (III3). Understanding the mechanics which leads to the result (III3), however, does not explain the physical implications of the unaccounted $1 / 3$ of the sum rule in the case of the rigid rotator. This is an important issue in view of the intimate relation of this sum rule to physical processes. It is investigated in the following.
A first glimpse into the question at hand is made possible by recalling the difference between a classical rotator and a quantal rotator. In the former case, a rigidly fixed value of $R$ implies that there is no radial momentum and the $P_{r}^{2}$ term disappears. In the quantal case, a "constant" value for $R$ means that the uncertainty in $R$ is much smaller than $R$, and hence the uncertainty in the radial momentum, or the expectation value of $P_{r}^{2}$, becomes very large; indeed, it tends to infinity in the limit of perfect rigidity. In consequence, radial excitations occur at very high energies in the quantal case, approaching infinitely high energies in the limit of perfect rigidity and zero uncertainty in $R$. We deduce from this and the earlier discussion that the apparently missing $1 / 3$ in the TRK sum rule for the rigid rotator is actually to be found at infinitely high energies.

At this point we need a specific example which illustrates these considerations. We present such an example in the following section.

\section{THE SPHERICAL $\delta$-SHELL POTENTIAL}

Consider a particle of mass $\mu$ in a $\delta$-shell potential $V(r)$ $=-g \delta(r-R)$. This potential has at most one bound state for each angular momentum value $l$ and a continuous spectrum above the "ionization" threshold. We shall show numerically that as $g$, the strength of the potential, increases, the bound-state energy spectrum will resemble that of a rigid rotator, and that $1 / 3$ of the strength in the TRK sum rule will be located at high energies in the continuum, i.e., above the dissociation threshold. For the perfectly rigid rotator, this threshold is obviously at infinite energy, and hence the missing sum rule strength will be located at infinity.

The Schrödinger equation for the radial wave function $R_{E_{l}}=u_{l}(r) / r$ in the case of the particle in a $\delta$-shell potential takes the form,

$$
\begin{aligned}
& \frac{d^{2} u_{l}(r)}{d r^{2}}-\frac{l(l+1)}{r^{2}} u_{l}(r)+\frac{2 \mu g}{\hbar^{2}} \delta(r-R) u_{l}(r) \\
& =-\frac{2 \mu}{\hbar^{2}} E_{l} u_{l}(r) .
\end{aligned}
$$

We shall next obtain solutions for this equation for both bound states and states in the continuum.

\section{A. Bound states}

We write the energy eigenvalue $E_{l}$ in terms of the wave number $\kappa_{l}$,

$$
E_{l}=-\left|E_{l}\right|=-\frac{\kappa_{l}^{2} \hbar^{2}}{2 \mu}
$$

and define a quantity $\lambda=2 \mu g / \hbar^{2}$; the radial equation becomes

$$
u_{l}^{\prime \prime}(r)-\frac{l(l+1)}{r^{2}} u_{l}(r)+\lambda \delta(r-R) u_{l}(r)=\kappa_{l}^{2} u_{l}(r)
$$

For $0<r<R$ and for $r>R$, this equation takes the form

$$
u_{l}^{\prime \prime}(r)-\frac{l(l+1)}{r^{2}} u_{l}(r)=\kappa_{l}^{2} u_{l}(r)
$$


This is the Bessel equation with solutions

$\frac{u_{l}(r)}{r}=\left\{\begin{array}{l}C_{l} j_{l}\left(i \kappa_{l} r\right), \quad 0<r<R \\ A_{l j_{l}}\left(i \kappa_{l} r\right)+B_{l} n_{l}\left(i \kappa_{l} r\right)=A_{l} h_{l}^{(1)}\left(i \kappa_{l} r\right), \quad r>R,\end{array}\right.$

where $j_{l}, n_{l}$, and $h_{l}^{(1)}=j_{l}+i n_{l}$ are the spherical Bessel, Neumann and Hankel (first kind) functions, respectively. For the case $0<r<R$ we take into account the fact that the solution must be regular at $r=0$.
Integrating Eq. (IV4) over the range $0<r<\infty$, we obtain a discontinuity in $u_{l}^{\prime}(r)$, the first derivative of $u_{l}(r)$, at $r=R$; that is

$$
u_{l}^{\prime}\left(R^{+}\right)-u_{l}^{\prime}\left(R^{-}\right)=-\lambda u_{l}(R)
$$

with $R^{ \pm}=R \pm \epsilon, \epsilon \rightarrow 0$. Dividing the above equation by $u_{l}(R)$, and using the continuity of this function at $r=R$, and Eq. (IV5), we find the result

$$
\left.\frac{A_{l}\left[r h_{l}^{(1)}\left(i \kappa_{l} r\right)\right]^{\prime}\left[r j_{l}\left(i \kappa_{l} r\right)\right]-A_{l}\left[r h_{l}^{(1)}\left(i \kappa_{l} r\right)\right]\left[r j_{l}\left(i \kappa_{l} r\right)\right]^{\prime}}{A_{l} r h_{l}^{(1)}\left(i \kappa_{l} r\right) .}\right|_{r=R}=-\lambda
$$

The numerator on the left-hand side of this equation is the Wronskian of the functions $r h_{l}^{(1)}\left(i \kappa_{l} r\right)$ and $r j_{l}\left(i \kappa_{l} r\right)$, and can be readily shown to be a constant independent of the value of $r$. Evaluating it at $r=0$, and substituting into Eq. (IV7) we find

$$
\frac{\left(\kappa_{l} R\right)}{(\lambda R)}=-\left(\kappa_{l} R\right)^{2} h_{l}^{(1)}\left(i \kappa_{l} R\right) j_{l}\left(i \kappa_{l} R\right) .
$$

We will solve this equation for the dimensionless product $\kappa_{l} R$ which defines the bound state energy $E_{l}$, Eq. (IV2), in terms of the dimensionless variable $y=\lambda R$.

\section{Normalization and energy eigenvalues}

The constants $C_{l}$ and $A_{l}$ in the wave function, Eq. (IV5) are determined by means of the wave function normalization condition and the continuity condition at $r=R$. We find the result

$$
\begin{aligned}
& C_{l}=c_{l} \kappa_{l}^{3 / 2}, \\
& A_{l}=a_{l} \kappa_{l}^{3 / 2} .
\end{aligned}
$$

The values of $c_{l}$ and $a_{l}$ for the angular momentum states $l=0$ and $l=1$, and for a range of values of $y=\lambda R$, indicative of potential strength, are shown in Table I.

Before we solve Eq. (IV8), it is useful to use it to derive the condition that an angular momentum state of quantum number $l$ be bound, for a given value of $\lambda R$. Clearly, this condition is satisfied for $\kappa_{l} R \rightarrow 0$. Employing the analytic expressions for spherical Bessel functions near the origin, we find that states $l$ are bound provided

$$
2 l+1 \leqslant \lambda R .
$$

Table I. Normalization constants, Eqs. (IV5) and (IV9), for the bound states $l=0$ and $l=1$, for a range of values of $\lambda R$.

\begin{tabular}{rcccc}
\hline \hline$\lambda R$ & $c_{0}$ & $a_{0}$ & \multicolumn{1}{c}{$c_{1}$} & $a_{1}$ \\
\hline 3.2 & -0.48466 & 4.86529 & 21.8283 & 0.86282 \\
4.8 & -0.19001 & 10.9836 & 0.75061 & 4.67521 \\
10.0 & -0.01348 & 148.411 & 0.02173 & 101.192 \\
16.0 & $-6.709-04$ & $2.981+03$ & $0.8820-03$ & $2.344+03$ \\
25.0 & $-7.453-06$ & $2.683+05$ & $0.8819-05$ & $2.298+05$ \\
\hline \hline
\end{tabular}

There is only one bound state for every value of $l$. With Eq. (IV10) in mind, we solve Eq. (IV8) numerically for a range of values of $\lambda R$. We show the results for $\kappa_{l} R, 0 \leqslant l \leqslant 4$, in Table II.

\section{Comparison of the relative distribution of energy states and of root-mean-square radii of the $\delta$-function potential and the rigid rotator}

Writing the energy $E_{l}$ of the rigid rotator in terms of a wave number $\kappa_{l}$, and using Eq. (III1), we find

$$
\frac{1}{2} \frac{\left[\left(\kappa_{l+1} R\right)^{2}-\left(\kappa_{l} R\right)^{2}\right]}{l+1}=1 \text { for the rigid rotator. }
$$

This gives a measure of the energy gap between successive angular momentum states in the case of the rigid rotator. At the same time, using the results for the $\delta$-function potential in Table II, we evaluate the left-hand side of Eq. (IV11) for this system also, and we find that as $\lambda R$ grows larger, its value approaches 1, i.e., the value in Eq. (IV11), rather quickly. Indeed, the two energy distributions, for the rigid rotator and for the $\delta$-function potential, become essentially identical when $\lambda R=25$. We show the results for the $\delta$-shell potential in Table III.

Additional information regarding how the $\delta$-function potential system approaches the rigid rotator is given by the values of the rms radius for the ground state in the former case, compared to the radius $R$ of the rigid rotator. Using the ground state wave function, Eqs. (IV5) and (IV9) and Table $\mathrm{I}$, for $l=0$, we evaluate both the rms radius $\sqrt{\left\langle r^{2}\right\rangle}$, and the

Table II. Solutions $\kappa_{l} R$ of Eq. (IV5) for the allowed bound states of angular momentum $l$, for a range of values of $\lambda R$.

\begin{tabular}{rccccc}
\hline \hline & \multicolumn{5}{c}{$\kappa_{l} R$} \\
\cline { 2 - 6 }$\lambda R$ & \multicolumn{1}{c}{$l=0$} & $l=1$ & $l=2$ & $l=3$ & $l=4$ \\
\hline 3.2 & 1.52409 & 0.478012 & & & \\
4.8 & 2.37942 & 1.83183 & & & \\
10.0 & 4.99977 & 4.78185 & 4.32325 & 3.54491 & 2.14704 \\
16.0 & 8.0 & 7.87087 & 7.60672 & 7.19396 & 6.60655 \\
25.0 & 12.50 & 12.4190 & 12.2553 & 12.0058 & 11.6651 \\
\hline \hline
\end{tabular}


Table III. Distribution of bound state energies for the $\delta$-function potential (similar to Eq. (IV11) for the rigid rotator).

\begin{tabular}{rllll}
\hline \hline & \multicolumn{4}{c}{$\frac{1}{2} \frac{\left[\left(\kappa_{l+1} R\right)^{2}-\left(\kappa_{l} R\right)^{2}\right]}{l+1}$} \\
\\
\cline { 2 - 4 }$\lambda R$ & & $l=1$ & $l=2$ & \\
\hline 3.2 & 1.047 & & & \\
4.8 & 1.153 & & & \\
10.0 & 1.065 & 1.043 & 1.021 & 0.995 \\
16.0 & 1.024 & 1.022 & 1.018 & 1.013 \\
25.0 & 1.009 & 1.009 & 1.009 & 1.008 \\
\hline \hline
\end{tabular}

quantity $\sqrt{\left\langle r^{2}\right\rangle-R^{2}}$. The latter reflects the "squeezing" of the wave function into a progressively narrower region as the potential becomes deeper. We display these results in Table IV. It is noted again that for $\lambda R=25$, the rms radius of the $\delta$-function potential is essentially equal to the radius $R$ of the rigid rotator.

\section{Contribution to the TRK sum rule from the bound states of the $\delta$-function potential}

Using the wave functions, Eq. (IV5), with the normalizations given by Eq. (IV9) and in Table I, we now evaluate the contribution to the TRK sum rule from bound-state excitations, Eq. (I7), in the case of the $\delta$-shell potential. The results are given in Table $\mathrm{V}$ for a range of values of the potential strength $\lambda R$ and are compared to the result for the rigid rotator which was found earlier to be $2 / 3$, Eq. (III3).

We reiterate the fact that the results in Tables III and IV show that the behavior of the $\delta$-shell potential system takes exactly the character of the quantized rigid rotator, in the neighborhood of $\lambda R=25$. This is now strengthened by the results in Table $\mathrm{V}$ which show that at that point, i.e., for $\lambda R=25$, the contribution to the TRK sum rule from the bound states of the $\delta$-shell potential is precisely the same as that for the rigid rotator, i.e., $\Sigma_{\text {bound states }} f_{n 0}=2 / 3$. Clearly, the nature of the TRK sum rule implies that there must be further excitations which will provide the additional $1 / 3$ required to saturate the sum rule. This must come from excitations to the continuum. While the rigid rotator has continuum states only at infinite energy, the $\delta$-shell potential has calculable continuum states at finite energy. We proceed, in the next subsection, to verify numerically that the missing strength in the TRK sum rule comes indeed from the continuum states.

Table IV. The rms radius for the ground state, and the confinement of the particle trapped in the $\delta$-function potential.

\begin{tabular}{|c|c|c|c|}
\hline$\lambda R$ & $\kappa_{0} R$ & $\frac{\sqrt{\left\langle r^{2}\right\rangle}}{R}$ & $\frac{\sqrt{\left\langle r^{2}\right\rangle-R^{2}}}{R}$ \\
\hline 3.2 & 1.52409 & 1.1552 & 0.57844 \\
\hline 4.8 & 2.37942 & 1.0571 & 0.34284 \\
\hline 10.0 & 4.49977 & 1.0101 & 0.14255 \\
\hline 16.0 & 8.0 & 1.0039 & 0.08854 \\
\hline 25.0 & 12.5 & 1.0016 & 0.05657 \\
\hline
\end{tabular}

Table V. Contribution to the TRK sum rule from bound states.

\begin{tabular}{rcc}
\hline \hline$\lambda R$ & $\Sigma_{\text {bound states }} f_{n 0}, \delta$-function potential & Rigid rotator \\
\hline 3.2 & 0.9042 & \\
4.8 & 0.8399 & \\
10.0 & 0.7142 & \\
16.0 & 0.6836 & $2 / 3$ \\
25.0 & $0.6729 \cong 2 / 3$ & \\
\hline \hline
\end{tabular}

\section{B. Continuum states}

The radial Eq. (IV1), with positive energy eigenvalues $E_{l}=\hbar^{2} k_{l}^{2} / 2 \mu$, has solutions

$\frac{v(r)}{r}=\left\{\begin{array}{l}\sqrt{\frac{2}{\pi}} D_{l j} j_{l}\left(k_{l} r\right), \quad 0<r<R \\ \frac{1}{2} \sqrt{\frac{2}{\pi}}\left[e^{2 i \delta_{l}} h_{l}^{(1)}\left(k_{l} r\right)+h_{l}^{(2)}\left(k_{l} r\right)\right], \quad r>R\end{array}\right.$,

where $h_{l}^{(2)}=j_{l}\left(k_{l} r\right)-i n_{l}\left(k_{l} r\right)$ is the Hankel function of the second kind. These wave functions satisfy the required completeness relation and run smoothly into the free particle wave functions in the limit $\lambda \rightarrow 0$. For the evaluation of the sum rule, Eq. (I7), we need only the $l=1$ wave functions, and so the rest of the discussion focuses on these states only.

The amplitude $D_{1}$ and the phase shift $\delta_{1}$ in Eq. (IV12) are found from the continuity of the radial wave function at $r=R$, and from an equation equivalent to (IV6) for the radial function $v(r)$. The results are as follows:

$$
D_{1}=\frac{1}{2}\left[e^{2 i \delta_{1}}\left(1+i \frac{n_{1}\left(k_{1} R\right)}{j_{1}\left(k_{1} R\right)}\right)+\left(1-i \frac{n_{1}\left(k_{1} R\right)}{j_{1}\left(k_{1} R\right)}\right)\right],
$$

and

$$
\tan \delta_{1}=\frac{\lambda k_{1} R^{2}\left|j_{1}\left(k_{1} R\right)\right|^{2}}{1+\lambda k_{1} R^{2} j_{1}\left(k_{1} R\right) n_{1}\left(k_{1} R\right)} .
$$

In the evaluation of Eq. (I7), the sum is replaced by an integral over the momentum $k_{1}$ of the $l=1$ state. As the strength of the potential, $\lambda R$, and the argument $k_{1} R$ of the Bessel functions appearing in this integral become very large, the numerical accuracy of the outcome suffers. We show the final results in Fig. 1, where we display the contribution to the sum rule from the bound states (shown also in Table V), and that from the states in the continuum, and the total contribution which, as expected, is complete; i.e.,

$$
\sum_{\text {bound states }}+\int_{\text {continuum }}=1
$$

when the two contributions are added. We stopped the numerical evaluation of the contribution from excitations to the continuum at about $\lambda R=12$, because numerical instabilities sap the reliability of results at higher values of $\lambda R$. But the point is adequately made, that the TRK sum rule is satisfied in the case of the spherical $\delta$-shell potential provided the contribution from the continuum states is taken into account. 


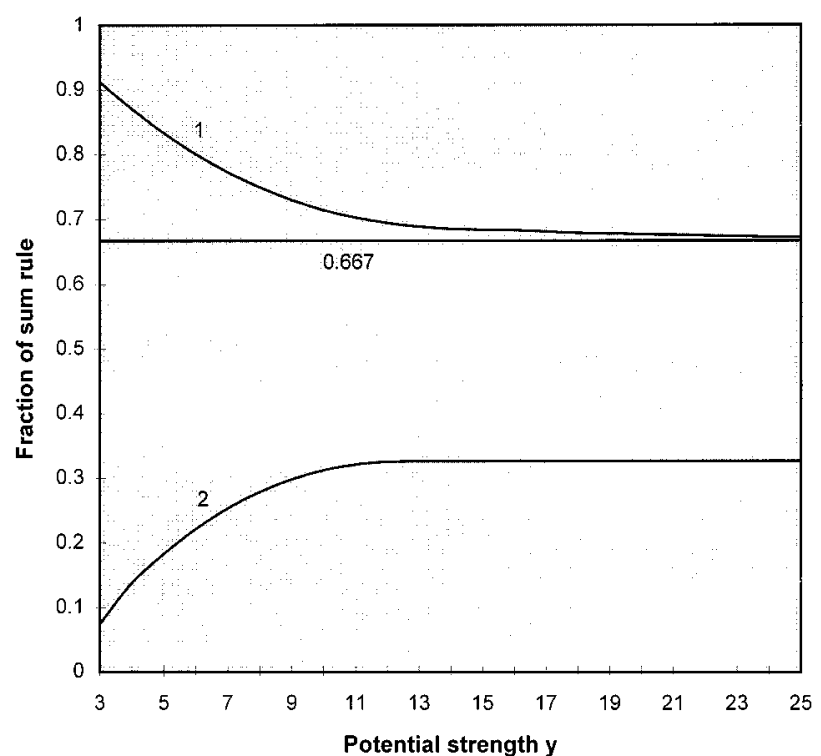

Fig. 1. The fraction of the TRK sum rule contributed by bound states (line 1 ) and continuum states (line 2) in the case of the $\delta$-shell potential, plotted versus the dimensionless variable $y=\lambda R$ which is a measure of the strength of the potential. At any point, the two contributions add up to 1 . The straight line shows the incomplete saturation, i.e., $2 / 3=0.667$, of the TRK sum rule in the case of the rigid rotator. For large values of the potential strength, i.e., $y \sim 25$, the contribution from the bound states of the $\delta$-shell potential becomes equal to that of the rigid rotator.

\section{ADDITIONAL SUM RULES}

It is worth extending the considerations of the previous sections to sum rules involving multipoles higher than the dipole. ${ }^{7}$ We define an operator

$$
Q_{\lambda}^{\mu}=r^{\lambda} Y_{\lambda, \mu}(\vartheta, \phi),
$$

where $Y_{\lambda, \mu}(\vartheta, \phi)$ with $\lambda \geqslant 1$ are the familiar spherical harmonics. Following a procedure similar to the one described earlier for the dipole sum rule, using the fundamental commutation relations between position and momentum variables and the completeness relation, we find the following sum rule in the case of a spherically symmetric ground state, $|0\rangle$ :

$$
\sum_{n}\left(E_{n}-E_{0}\right)\left|\left\langle n\left|Q_{\lambda}^{\mu}\right| 0\right\rangle\right|^{2}=\frac{\hbar^{2}}{2 m} \frac{\lambda(2 \lambda+1)}{4 \pi}\left\langle 0\left|r^{2 \lambda-2}\right| 0\right\rangle .
$$

It is interesting to examine this sum rule also for the rigid rotator of length $R$. The energy $E_{n}$ is given by Eq. (III1), and the matrix element on the left-hand side of Eq. (V2) is $\left\langle l, m\left|Q_{\lambda}^{\mu}\right| 0\right\rangle=\delta_{l, \lambda} \delta_{m, \mu} R^{l} / \sqrt{4 \pi}$, where we have substituted $\langle l, m|$ for $\langle n|$. Hence the left-hand side of Eq. (V2) is $\left(\hbar^{2} / 2 m\right) /(\lambda(\lambda+1) / 4 \pi) R^{2 \lambda-2}$. Furthermore, with $\left\langle 0\left|R^{2 \lambda-2}\right| 0\right\rangle=R^{2 \lambda-2}$, the right-hand side of (V2) takes the form $\left(\hbar^{2} / 2 m\right)(\lambda(2 \lambda+1) / 4 \pi) R^{2 \lambda-2}$. Comparing these two results, we find that the fraction of the sum rule limit, Eq. (V2), exhausted by the bound states of the rigid rotator is $(\lambda+1) /(2 \lambda+1)$. Obviously, for $\lambda=1$, this corresponds to the previously found result of $2 / 3$ for the dipole case, Eq. (III3). For quadrupole transitions, $\lambda=2$, this fraction is $3 / 5$, and, in general, $\frac{\lambda+1}{2 \lambda+1} \rightarrow \frac{1}{2}$ for $\lambda \gg 1$.

Hence, for high values of $\lambda$ only half the strength of the sum rule, Eq. (V2), is contained in the spectrum of the rigid rotator.

Equally interesting is the case of the monopole sum rule where we show that the entire sum rule strength is missing. The monopole operator is $\bar{r}^{2}$, and the corresponding sum rule takes the form

$$
\sum_{n}\left(E_{n}-E_{0}\right)\left|\left\langle n\left|\bar{r}^{2}\right| 0\right\rangle\right|^{2}=2 \frac{\hbar}{m}\left\langle 0\left|\bar{r}^{2}\right| 0\right\rangle .
$$

For the rigid rotator, the right-hand side of Eq. (V3) is $2 \hbar^{2} / m R^{2}$, while the left-hand side is zero for the ground state, $n=0$, because of Eq. (III1), and remains zero for $n>0$ due to angular momentum conservation. Hence, the contribution to the monopole sum rule from all the rotational states of the rigid rotator is zero, and the entire strength of the sum rule is to be found at infinite energies. This is a striking result, but hardly surprising. The excitations induced by the monopole operator $\bar{r}^{2}$ do not involve changes of shape or orientation, but changes of size only. These, however, require infinite energy due to the rigidity of the rotator.

\section{CONCLUSION}

We showed through a direct calculation that the TRK sum rule for dipole excitations by photon absorption is apparently not saturated in the case of the rigid rotator. All the energy states of the rigid rotator are bound and their contribution to the TRK sum rule, i.e., the left-hand side of Eq. (I7), is only $2 / 3$ of the expected value. At the same time, we noticed that in a spherically symmetric Hamiltonian, the centrifugal term in the kinetic energy contributes $2 / 3$ of the sum rule value, while the radial momentum term $P_{r}^{2} / 2 m$ contributes the additional $1 / 3$. In the case of a perfectly rigid rotator, the radial momentum term is missing and, consequently, $1 / 3$ of the sum rule should also be missing. Hence, the result of the direct calculation was confirmed.

In the quantum regime, however, the radial momentum term $P_{r}^{2} / 2 m$ in the rotator Hamiltonian must be very large, instead of being zero, due to the uncertainty principle. We deduce that the apparently missing $1 / 3$ strength of the TRK sum rule, instead of being truly absent, will be found in the very high energy region of the spectrum.

Indeed, we illustrated this fact by examining a threedimensional system under the action of a $\delta$-shell potential. The rigid rotator is the limiting case of this system when the potential becomes very strong. As expected, we found that as the rigidity of the potential was increased, e.g., at $\lambda R \sim 25$ (see Tables III-V), the behavior of the system approached that of the rigid rotator, and the TRK sum rule was satisfied by contributions from bound and continuum states, providing $2 / 3$ and $1 / 3$ of the sum rule, respectively, as illustrated in Fig. 1 . We conclude that in the case of the rigid rotator as well, the balance of $1 / 3$ is contributed to the TRK sum by states in the continuum. The difference is that for the rigid rotator these states are at infinite energy.

\section{ACKNOWLEDGMENT}

EH was supported in part by NSF Grant No. PHY9213738. 
a)Present address: Echo Systems Center, Marine Biological Laboratory, 7 MBL Street, Woods Hole, MA 02543.

${ }^{1}$ W. Thomas, "Über die Zahl der Dispersionelektronen die einem stationären Zustand Zugeordnet sind,'” Naturwissenschaften 13, 627-628 (1925); F. Reiche and W. Thomas, "Über die Zahl der Dispersionelektronen die einem stationären Zustand Zugeordnet sind," Z. Phys. 34, 510-525 (1925); W. Kuhn, “Über die Gesamtstärke der von einem Zustande ausgehenden Absorptionslinien," ibid. 33, 408-412 (1925).

${ }^{2}$ E. Merzbacher, Quantum Mechanics (Wiley, New York, 1961), p. 446.

${ }^{3}$ H. A. Bethe and R. W. Jackiw, Intermediate Quantum Mechanics (Benjamin, New York, 1968), 2nd ed., p. 148.

${ }^{4} \mathrm{G}$. Baym, Lectures in Quantum Mechanics (Benjamin, Redding, 1976), p. 287.
L. D. Landau and E. M. Lifshitz, Relativistic Ouantum Theory (Pergamon, Oxford, 1961) Eq. (60.15), p. 193.

${ }^{6}$ W. Currie, "The Thomas-Reiche-Kuhn Sum Rule and Distribution of the Quantal Oscillator Strengths," senior thesis, 1983; some of the material discussed here was presented in this thesis which was submitted in partial fulfillment of the Sc.B. degree requirement at Brown University.

${ }^{7}$ T. J. Deal and S. Fallieros, "Models and Sum Rules for Nuclear Transition Densities,' Phys. Rev. C 7, 1709-1712 (1973); S. Fallieros, in Nuclear Structure Studies Using Electron Scattering and Photoreactions, edited by A. Ui (Laboratory of Nuclear Science, Tohoku University, 1972), Vol. 5, pp. $185-198$. 